\title{
Antiviral and virucidal activities of sulphated polysaccharides against Japanese encephalitis virus
}

\author{
Nor Rashid, N. ${ }^{1}$, Yusof, R. ${ }^{1}$ and Rothan, H.A. ${ }^{2 *}$ \\ ${ }^{1}$ Department of Molecular Medicine, Faculty of Medicine, University of Malaya, 50603 Kuala Lumpur, Malaysia \\ ${ }^{2}$ Department of Biology, College of Arts and Sciences, Georgia State University, Atlanta, GA, United States \\ *Corresponding author e-mail: hrothan@gsu.edu \\ Received 25 May 2019; received in revised form 16 July 2020; accepted 17 July 2020
}

\begin{abstract}
Japanese encephalitis virus (JEV), a member of the family Flaviviridae, causes severe neurological disorders in humans. JEV infections represent one of the most widely spread mosquito-borne diseases, and therefore, it has been considered as an endemic disease. An effective antiviral drug is still unavailable to treat JEV, and current drugs only provide supportive treatment to alleviate the symptoms and stabilize patients' conditions. This study was designed to evaluate the antiviral activity of the sulphated polysaccharides "Carrageenan," a linear sulphated polysaccharide that is extracted from red edible seaweeds against JEV replication in vitro. Viral inactivation, attachment, and post-infection assays were used to determine the mode of inhibition of Carrageenan. Virus titters after each application were evaluated by plaque formation assay. MTT assay was used to determine the $50 \%$ cytotoxic concentration (CC50), and ELISA-like cell-based assay and immunostaining and immunostaining techniques were used to evaluate the 50\% effective concentration (EC50). This study showed that Carrageenan inhibited JEV at an EC50 of $15 \mu \mathrm{g} / \mathrm{mL}$ in a dose-dependent manner with CC50 more than $200 \mu \mathrm{g} / \mathrm{mL}$ in healthy human liver cells (WRL68). The mode of inhibition assay showed that the antiviral effects of Carrageenan are mainly due to their ability to inhibit the early stages of virus infection such as the viral attachment and the cellular entry stages. Our investigation showed that Carrageenan could be considered as a potent antiviral agent to JEV infection. Further experimental and clinical studies are needed to investigate the potential applications of Carrageenan for clinical intervention against JEV infection.
\end{abstract}

\section{INTRODUCTION}

Flaviviruses infection has been disseminated across many countries in tropical countries (Mackenzie et al., 2004; Rothan et al., 2018). Japanese encephalitis virus (JEV) infections represent one of the most widely spread mosquito-borne diseases, and therefore, it has been considered as an endemic disease, predominantly occurs in South-East Asia and the Western Pacific regions. The infection is mainly caused by the transmission of JEV to humans through mosquitoes from the Culex genus, especially Culex tritaeniorhynchus. The symptom of JEV infection ranges from mild to severe manifestation. Most infected individuals only experience mild symptoms such as fever and headache. However, some $(<1 \%)$ might develop severe clinical symptoms such as high fever, spastic paralysis, and coma (Mackenzie et al., 2004; Hills et al., 2009). There are approximately 44,000 new cases of JEV with clinical symptoms per year with a $30 \%$ mortality rate, while $30-50 \%$ of survivors will suffer permanent neurological impairment (Wang et al., 2015; Hills et al., 2009). Although vaccines for JEV are available, long term protective effects is still not known, and treatment usually requires at least one booster dose after 12 months of vaccination. This requirement is highly inconvenient and usually leads to low public compliance. An effective antiviral drug is still unavailable to 
treat JEV, and current drugs only provide supportive treatment to alleviate the symptoms and stabilize patients' conditions (Wang et al., 2015; Hills et al., 2009). As such, the development of active antiviral agents for JEV is necessary to address the unmet medical needs.

Natural compounds show considerable antiviral activities through direct binding with viral proteins or cell membrane receptors (Rothan et al., 2012; Rothan et al., 2014). Carrageenan is sulphated highmolecular-weight polysaccharides extracted from red edible seaweeds and widely used in the food industry. Chemically, Carrageenan is made up of repeating galactose units joined by alternating $\alpha-1,3$ and $\beta-1,4$ glycosidic linkages. Sulphated polysaccharide was found to be very potent and selective inhibitors of Human Immunodeficiency Virus-1 (HIV-1) properties (Rothan et al., 2014). These effects are believed to be the result of direct binding to HIV glycoprotein 120 (gp120) on the surface of the viral particle that, in turn, inhibit the entry of HIV-1 into human cells (Rothan et al., 2014). In this study, we report the antiviral properties and cytotoxicity of Carrageenan towards JEV on WRL68 cells. The mechanisms of the inhibitory effects of Carrageenan were also investigated in order to get a better understanding of Carrageenan as a potential antiviral agent for combating JEV infections.

\section{MATERIALS AND METHODS}

The stocks of JEV were prepared in C6/36 cells, and viral titers were determined via plaque assay using Vero cells (ATCC; Rockville, MD, USA). The cells were cultured in complete Dulbecco's modified Eagle's medium (DMEM) supplemented with 10\% fetal bovine serum (FBS) as a growth medium or $2 \%$ FBS as maintenance medium. Iota Carrageenan and heparin were purchased from Sigma (Sigma Aldrich) and dissolved in $5 \%$ DMSO. The final concentration of DMSO was lower than $1 \%$ in all cell culturebased assays.

Healthy liver cells (WRL68, $1 \times 104$ cells per well of a 96 -well plate) were treated with increasing concentrations $(12.5,25,50,100$, and $200 \mu \mathrm{g} / \mathrm{ml}$ ) of Carrageenan or heparin as a positive control for $72 \mathrm{~h}$. The cytotoxic effect of the test compound was determined based on MTT (3-(4,5-dimethylthiazol 2-yl)-2,5diphenyltetrazolium bromide) assay, as described previously (Rothan et al., 2015).

A 10-fold serial dilution of culture supernatant of JEV infected cells was added to fresh Vero cells grown in 6-well plates $(1.2 \times 106$ cells) and incubated for $1 \mathrm{~h}$ at $37^{\circ} \mathrm{C}$. The cells were overlaid with DMEM containing $1.1 \%$ methylcellulose. Viral plaques were stained with crystal violet dye after a 5-day incubation period. Virus titers were calculated according to the following formula: Titer (p.f.u./ml) = number of plaques $\times$ volume of the diluted virus added to the well $\times$ dilution factor of the virus used to infect the well in which the plaques were enumerated (Rothan et al., 2013).

Subsequently, the cells were seeded into 24 -well plates $(1 \times 105$ cells per well $)$ for $24 \mathrm{~h}$ at $37^{\circ} \mathrm{C}$ and $5 \% \mathrm{CO}$. JEV culture supernatant was separately added to the cells at an M.O.I. of 1, followed by incubation for two $\mathrm{h}$ with gentle shaking every $15 \mathrm{~min}$ to achieve optimal virus-to-cell contact. The cells were washed twice with phosphatebuffered saline (PBS) after removing the virus culture supernatant. Subsequently, the new complete DMEM containing $25 \mu \mathrm{g} / \mathrm{ml}$ of Carrageenan or heparin was added to the cultures and further incubated for $72 \mathrm{~h}$. Viral titers were evaluated using plaque formation assays.

Virus inactivation assay was performed to assess the ability of Carrageenan to inactivate JEV, and prevent subsequent infections directly. WRL68 cells were seeded in 24 -well plates $(1 \times 105$ cells/well $)$ for $24 \mathrm{~h}$. The test compound $(25 \mu \mathrm{g} / \mathrm{ml})$ was mixed with the virus culture supernatant (M.O.I of 2) and incubated at $37^{\circ} \mathrm{C}$ for $1 \mathrm{~h}$ in a separate tube. Following this, the mixture was then inoculated onto the WRL68 cells with gentle shaking every $15 \mathrm{~min}$ for $2 \mathrm{~h}$. The virus and test compound mixture were then removed, and the cells were washed three times with PBS to remove any residual virus. Then, fresh complete DMEM was added, and the cultures were further incubated for $72 \mathrm{~h}$ at 
$37^{\circ} \mathrm{C}$ supplemented with $5 \%$ CO2. The viral titers of JEV suspension were then determined via plaque formation assays.

Viral attachment assay was performed to show the ability of Carrageenan to inhibit JEV entry into host cells. WRL68 cells were grown in 24 -well plates $(1 \times 105$ cells/well $)$ for $24 \mathrm{~h}$. Cell culture media were removed and the cells were then washed three times with PBS. New media containing virus and the test compound $(25 \mu \mathrm{g} / \mathrm{ml})$ mixture were separately added and the cells were incubated for one $\mathrm{h}$ at $4^{\circ} \mathrm{C}$. Subsequently, the media was removed and the cells were washed extensively with cold PBS to remove the unabsorbed virus. The cells were overlaid with DMEM containing 1.1\% methylcellulose and incubated for five days. Viral titers were quantified by counting infectious centers (Rothan et al., 2019).

The cells were seeded in 24-well plates $(1 \times 105$ cells/well $)$ for $24 \mathrm{~h}$ for post infection treatment. Cell culture media were removed and the cells were then washed three times with PBS. New media containing virus (M.O.I of 2) was added and incubated at $37^{\circ} \mathrm{C}$ with gentle shaking every $15 \mathrm{~min}$ for two $\mathrm{h}$. The virus was then removed by washing the cells twice with PBS followed by the addition of new complete DMEM containing $(25 \mu \mathrm{g} / \mathrm{ml})$ of the test compound. The cells were then incubated for $72 \mathrm{~h}$. The viral titers of JEV were then determined by plaque formation assays.

Finally, immunofluorescence assay was carried out with specific antibodies. Vero cells grown on coverslips were infected with JEV (M.O.I. of 2) in the presence or absence of Carrageenan (10, 20 and $30 \mu \mathrm{g} / \mathrm{ml})$. At $72 \mathrm{~h}$ post-infection, cell monolayers were washed with cold PBS and fixed in methanol for 15 min at $-20^{\circ} \mathrm{C}$ for cytoplasmic immunofluorescence. Indirect staining was carried out by using anti-JEV mouse antibodies and fluorescein (FITC)-labeled goat anti-mouse IgG (Rothan et al., 2014).

All assays were performed in triplicates, and the statistical analyses were performed using GraphPad Prism version 5.01 (GraphPad Software, San Diego, CA). P values $<0.05$ were considered significant. The error bars are expressed as $\pm \mathrm{SD}$.

\section{RESULTS}

The potential cytotoxicity effect of Carrageenan was evaluated using WRL68 cells and heparin was employed as a positive control. The $50 \%$ cytotoxic concentration (CC50) was higher than $200 \mu \mathrm{g} / \mathrm{ml}$ for both Carrageenan and heparin. The maximal non-toxic dose (MNTD) value was approximately $25 \mu \mathrm{g} / \mathrm{ml}$ and showed at least 95\% cell viability. Following this, all subsequent in vitro cell culture experiments were carried out using doses of less than $25 \mu \mathrm{g} / \mathrm{ml}$ of the potential compounds (Fig. 1A and 1B).

Liver cell lines (WRL68) showed normal morphology before infection with JEV virus. After JEV-infection, the cells showed various cytopathic effects (CPE). Treatment with carrageenan or heparin showed approximately normal monolayer sheets with lessen CPE (Fig. 2). Then, the antiviral activity of carrageenan against JEV was investigated by plaque formation assay. Our results showed that carrageenan acts by inhibiting viral entry into WRL68 cells, and the inhibitory strength of carrageenan towards JEV was at a similar level as heparin. The results obtained from the direct virus inactivation assay (Fig. 3A) demonstrated that carrageenan had a significant inhibitory effect against JEV $(6.7 \pm 2.2$ p.f.u./ml) compared to untreated cells $(19.6 \pm 1.8$ p.f.u./ $\mathrm{ml}$ ) while no significant difference was observed for heparin as a positive control $(7.2 \pm 2.6$ p.f.u./ml) compared to carrageenan (t-test, $\mathrm{p}<0.001$ ). Similarly, carrageenan also showed significant inhibitory effects at the viral attachment stage with $4.7 \pm 3.1$ p.f.u. $/ \mathrm{ml}$ inhibition for JEV infection compared to untreated cells $(18.2 \pm 2.4$ p.f.u./ml) and no significant difference compared to heparin (3.8 \pm 2.9 p.f.u. $/ \mathrm{ml}$ ) as a positive control (t-test, $\mathrm{P}<0.01)$ as presented in Figure 3B. We then incubated the WRL68 cells with JEV at $37^{\circ} \mathrm{C}$ for two $h$ and subsequently treated the cells with the test compounds to determine their inhibitory effects against viral replication post-infection. The post-infection treatment showed that carrageenan reduced viral titter to $13.4 \pm 2.2$ p.f.u./ml compared to untreated cells (21.7 \pm 2.6 p.f.u./ml ). For post-infection assay heparin had showed similar inhibition 


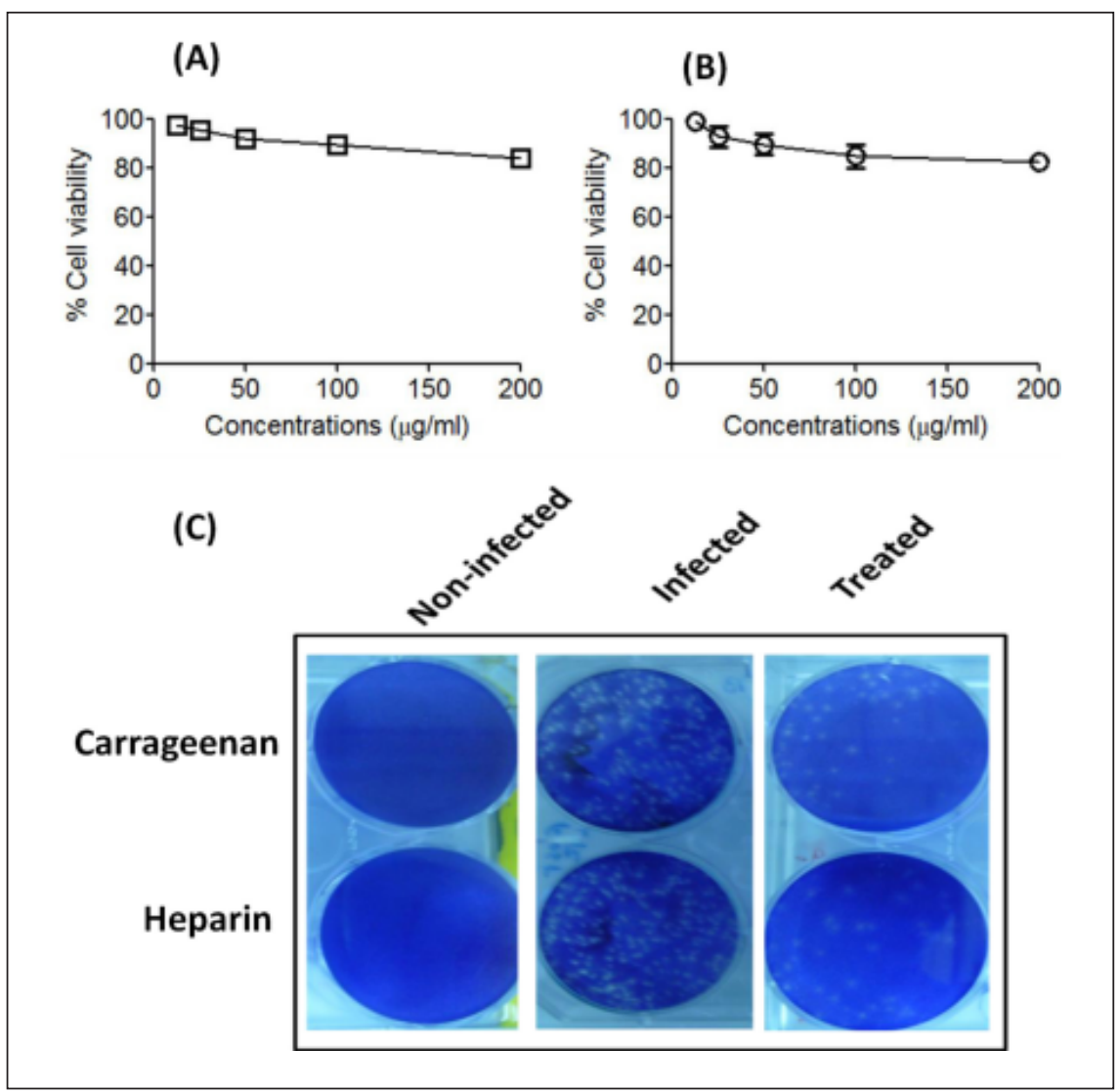

Figure 1. Cell viability of WRL68 cells after treatment with carrageenan and the positive control, heparin and test the antiviral activity by plaque assay.

(A) The $\mathrm{CC}_{50}$ value concentration was higher than $200 \mu \mathrm{g} / \mathrm{ml}$ for carrageenan (B) The $\mathrm{CC}_{50}$ value concentration was higher than $200 \mathrm{\mu g} / \mathrm{ml}$ for heparin. The MNTD value was approximately at $25 \mu \mathrm{g} / \mathrm{ml}$ for both compounds that showed at least $95 \%$ cell viability. (C) Determination of viral inhibition by the test compounds using plaque formation assay. The cells were pre-treated separately with $25 \mu \mathrm{g} / \mathrm{ml}$ of each test compounds and infected with JEV at MOI of 1 . After $72 \mathrm{hrs,} \mathrm{a} \mathrm{10-fold} \mathrm{serial} \mathrm{dilution} \mathrm{of} \mathrm{culture} \mathrm{supernatant} \mathrm{of} \mathrm{JEV-}$ infected cells was added to fresh Vero cells grown in 6 -well plates $\left(1.2 \times 10^{6}\right.$ cells). Viral plaques were stained with crystal violet dye after 5 days of incubation. MNTD=Maximal non-toxic dose.

towards JEV $(13.2 \pm 3.8$ p.f.u./ml) infection (t-test, $\mathrm{P}<0.05$ ) (Fig. 3C). Moreover, treatment with carrageenan also showed significant protective effects which retained the viability of infected cells to approximately $80 \%$ (t-test, $\mathrm{p}<0.01$ ) compared to untreated cells that showed cell viability of 40\% (Fig. 3D).

Our investigations showed Carrageenan increased inhibition potentials against JEV; we next characterized the antiviral activity of Carrageenan against JEV using an ELISAlike cell-based assay (Fig. 4). Our results demonstrated that Carrageenan inhibited JEV replication in WRL68 cells in a dosedependent manner. The 50\% effective concentration (EC50) for Carrageenan was approximately $15 \mu \mathrm{g} / \mathrm{ml}$, which is similar to the positive control heparin (Fig. 4). The immunostaining experiment showed that the concentration level of virus particles was considerably decreased after applying increased concentrations from $10 \mu \mathrm{g} / \mathrm{ml}$ to $30 \mu \mathrm{g} / \mathrm{ml}$ of Carrageenan (Fig. 5). 


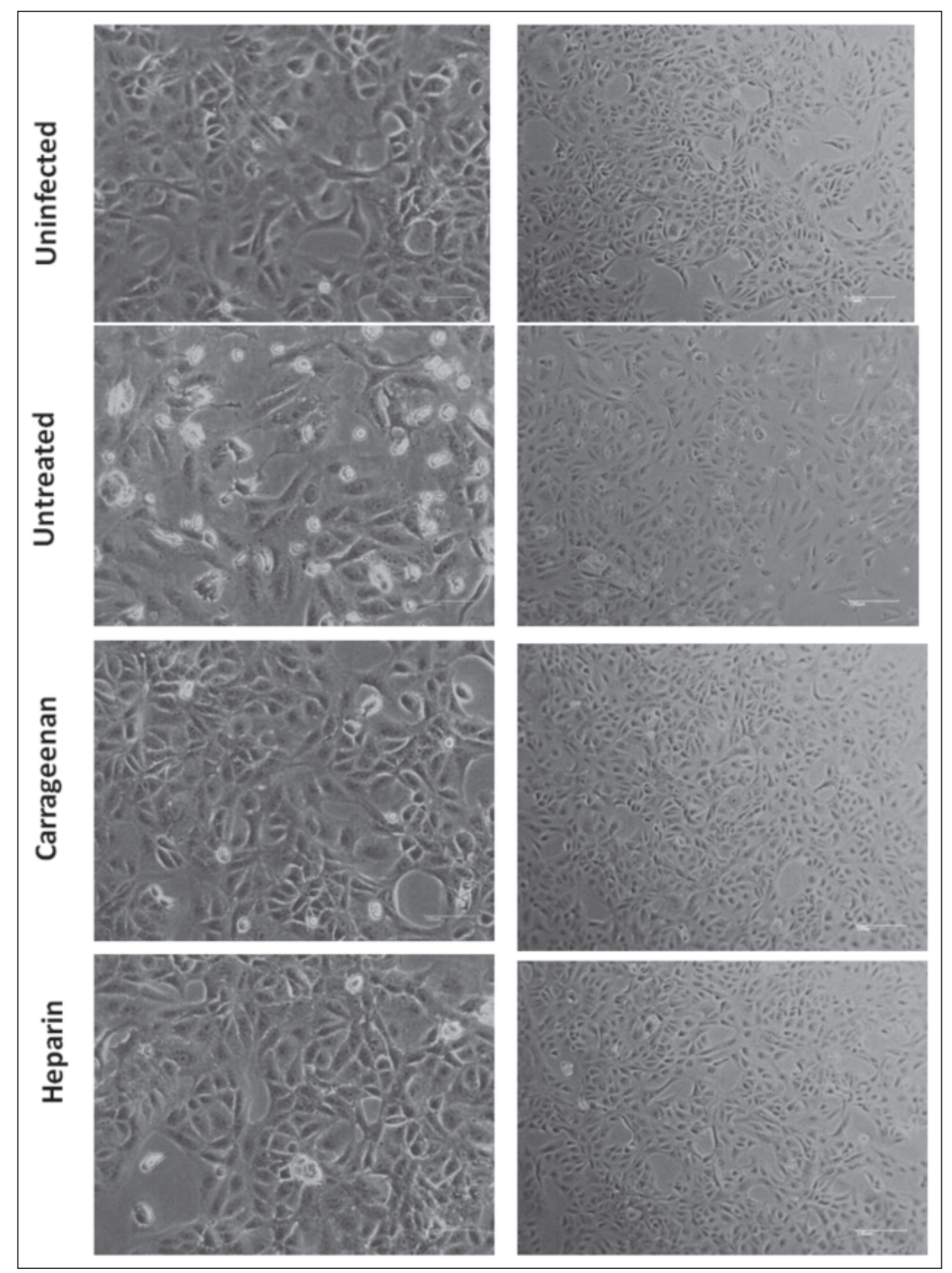

Figure 2. Morphological changes of JEV-infected cells treated with Carrageenan or heparin.

Uninfected cells showed normal cell morphology of normal liver cell lines WRL68. The untreated cells morphology post-infection showed various cytopathic effects (CPE). Treatment with carrageenan or heparin showed approximately normal monolayer sheet with lessen CPE. Cell morphology was examined using an inverted microscope at $200 \times$ magnification. 


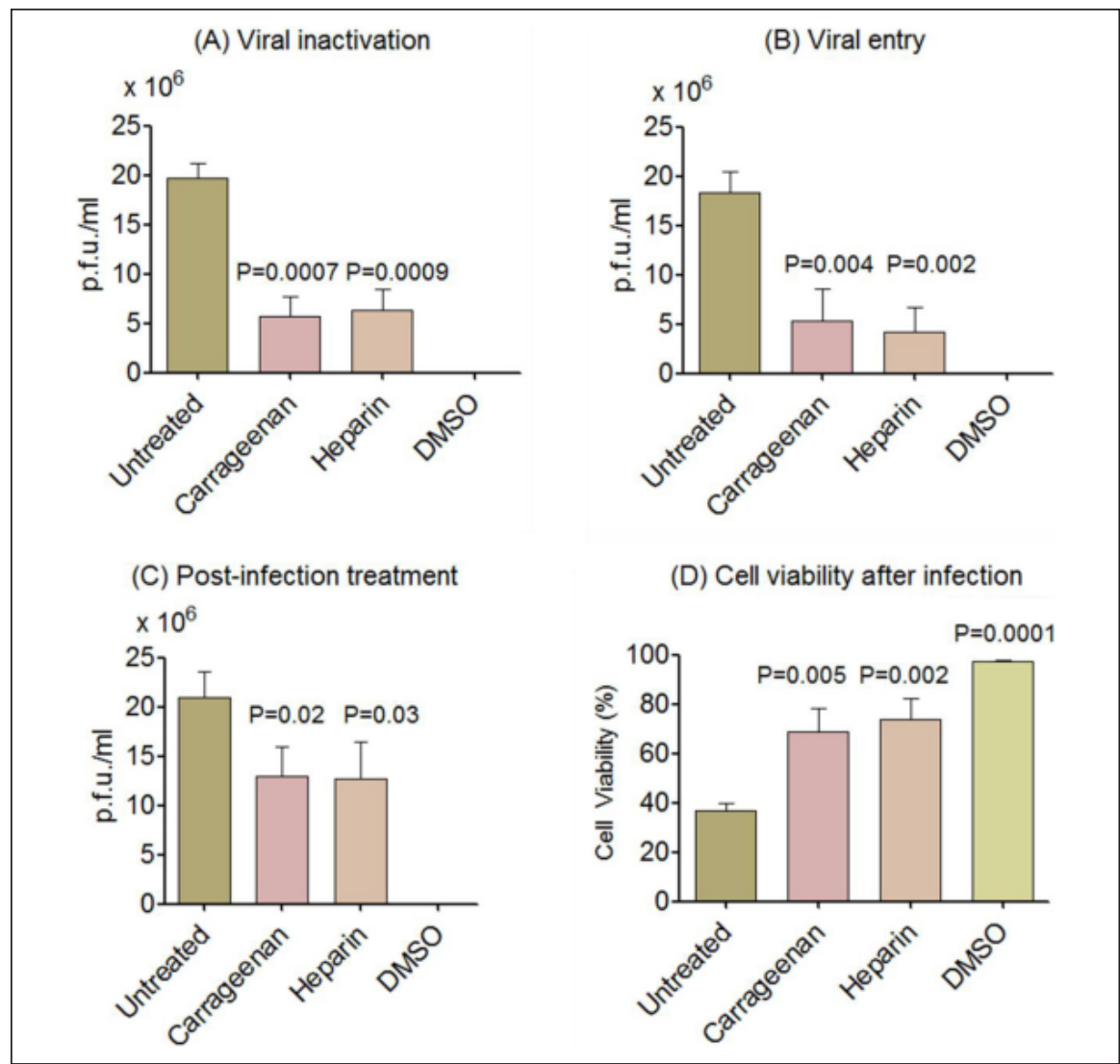

Figure 3. Mode of inhibition of carrageenan against JEV replication.

(A) Viral inactivation assay showed significant viral inhibition when viral particles were treated with carrageenan compared to untreated cells. Carrageenan showed similar inactivation potential compared to the positive control heparin. (B) Viral attachment assay showed that carrageenansignificantly inhibited virus attachment to target cells that was similar to heparin. (C) Post-infection treatment showed that the inhibitory effect of carrageenan against JEV was significant but at lower extent compared to the antiviral activity against viral entry (t-test $\mathrm{p}<0.01)$. Moreover, treatment with carrageenan also showed significant protective effects which retained the viability of infected cells to more than $75 \%$ (t-test, $\mathrm{p}<0.01$ ).

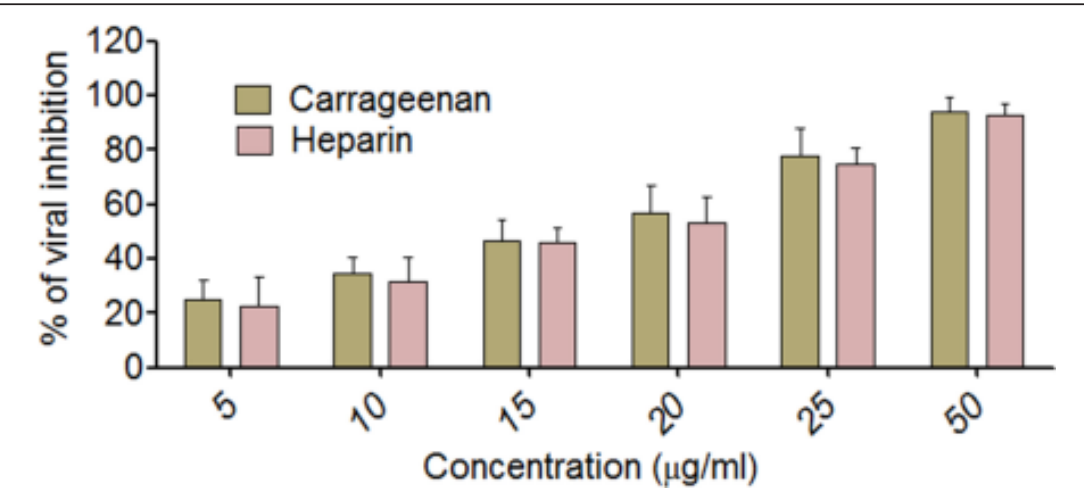

Figure 4. Viral inhibition of JEV in WRL68 cells by carrageenan.

Carrageenan inhibited JEV replication in WRL68 cells in a dose-dependent manner. The EC $_{50}$ value for carrageenan was approximately $15 \mu \mathrm{g} / \mathrm{ml}$ which is approximately similar to the positive control heparin. 


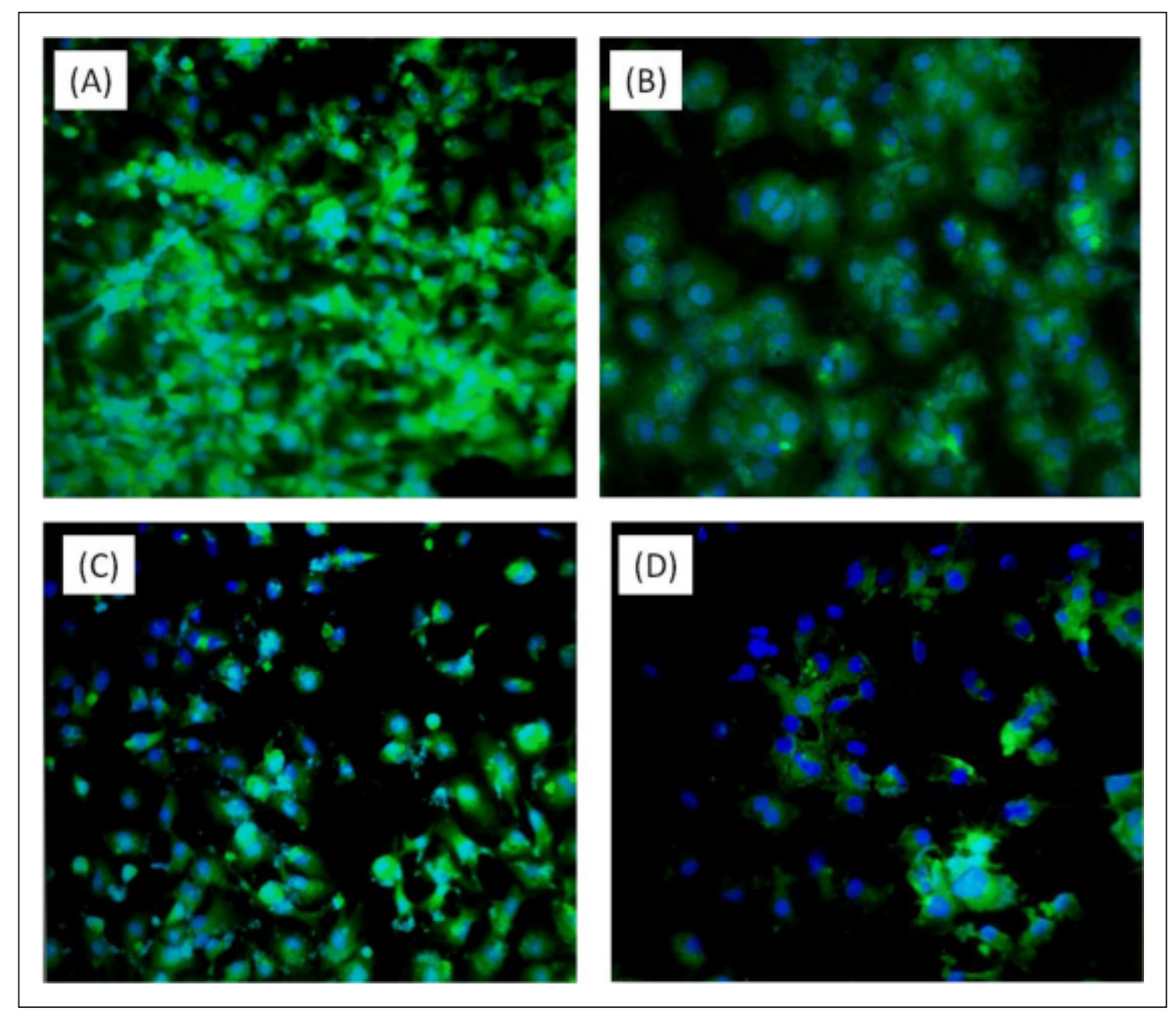

Figure 5. The effect of carrageenan in reducing viral particles.

The cells were grown on cover slips in 6-well plates and infected with JEV. The cells were then treated with increasing concentrations of carrageenan (A, $0 \mu \mathrm{g} / \mathrm{ml} ; \mathbf{B}, 10 \mu \mathrm{g} / \mathrm{ml} ; \mathbf{C} 20 \mu \mathrm{g} / \mathrm{ml} ; \mathbf{D}, 30 \mu \mathrm{g} / \mathrm{ml}$ ) for $72 \mathrm{~h}$. The fixed cells were treated with anti-JEV mouse antibodies and then treated with fluorescein (FITC)-labeled goat anti-mouse IgG. The results showed that the inhibitory effects were dependent on increasing concentrations of carrageenan.

\section{DISCUSSION}

In response to the urgent need for effective anti-JEV drugs, this study was proposed to identify and evaluate new inhibitors for the treatment of JEV infections. Best of our knowledge, the potential of the use of Carrageenan to inhibit JEV has not been previously investigated. Our studies have indeed shown that Carrageenan demonstrated minimal cytotoxicity while exhibiting significant inhibitory effects towards JEV $($ EC50 $=15 \mu \mathrm{g} / \mathrm{ml})$ infections in the normal human liver cell (WRL68), suggesting the potential of Carrageenan as an antiviral agent to combat JEV infections.
In all experiments, Heparin was used as a positive control.

In this study, three assays were performed to elucidate the antiviral mechanisms of Carrageenan. Our results indicate that Carrageenan inhibits JEV mainly through its direct virucidal effects and interference within the early stages of viral infection, such as viral attachment and cellular entry. We postulated that the direct antiviral activity of Carrageenan might be due to their ability to interact with the virus envelop glycoprotein, particularly the flavivirus $\mathrm{E}$ protein, which is responsible for JEV adsorption through the host membrane (Smit et al., 2011; Wang et al., 2015; Schmidt 
et al., 2010). Besides, Carrageenan also showed a significant inhibitory effect in the viral attachment assay. In contrast to the direct virucidal activity, the prevention of viral attachment might be attributed to the ability of Carrageenan to compete with both viruses for the cellular receptors (i.e. heparin sulphate) required for cellular entry and subsequent infections (De La Guardia et al., 2014).

On the other hand, in the post-infection assay, Carrageenan showed lower but significant antiviral activities towards JEV infection. It has been found that heparan sulphate (HS) is involved in the first interaction with $\mathrm{E}$ glycoprotein to initiate a flavivirus multiplication cycle in different types of vertebrate cells (Chen et al., 2007). $\mathrm{HS}$ is a member of highly sulphated glycosaminoglycans (GAGs), which is very abundant on the surface of most mammalian cells and serves as a receptor for many microbial agents, including bacteria, parasites, and viruses (Spillmann et al., 2001). For DENV, the interaction with HS is unusual, owing to its specificity for a highly sulphated form of HS[19]. Therefore, the polysaccharide, curdlan sulphate showed a potential antiviral activity through interfering with the cellular entry of DENV (Ichiyama et al., 2013).

Our studies also showed that Carrageenan exhibited differing levels of antiviral strength towards JEV, in each of the assays that have been carried out. Although the underlying reasons are still unclear, we suspect it may be due to the variability in the viral glycoproteins and cellular receptors, which are crucial for each virus to establish infection. On the other hand, Heparin has shown similar inhibitory activity in the postinfection assay. Although the potential use of Carrageenan to inhibit JEV, there are still some limitations that have not been addressed in this study. Researches on polysaccharides as antivirals has shown that the antiviral properties of polysaccharides are highly dependent on the structural complexity (i.e. number and position of the branches, branch length and chemical modifications of the polysaccharides) (Jiao et al., 2011; Ghosh et al., 2009). However, it is difficult for researchers to accurately characterize and purify a particular type of polysaccharide from the extracts of natural sources, which also remains as an inherent limitation in this study. To this end, further research is still needed to elucidate the exact mechanism of inhibition by Carrageenan and its association with the JEV virus replication cycle. Downstream studies with suitable animal models are perhaps required to evaluate the reproducibility of the inhibitory effects of Carrageenan in vivo.

\section{CONCLUSION}

Our study has successfully demonstrated the inhibition of JEV replication by Carrageenan with minimal cytotoxicity effects. Carrageenan could potentially be a promising broad-spectrum antiviral agent targeting the flavivirus family, and thus warrants further investigation.

Acknowledgements. This study was funded by the Ministry of Higher Education through the Trans Disciplinary Research Grant Scheme (TRGS 2014-2).

\section{REFERENCES}

Chen, Y., Maguire, T., Hileman, R.E., Fromm, J.R. \& Esko, J.D. (1997). Dengue virus infectivity depends on envelope protein binding to target cell heparan sulfate. Nature Medicine 3: 866-871.

Chen, J. \& Seviour, R. (2007). Medicinal importance of fungal beta-(1->3), (1->6)-glucans. Mycological Research 111: 635-652.

De La Guardia, C. \& Lleonart, R. (2014). Progress in the identification of dengue virus entry/fusion inhibitors. Biomed Research International 2014: 825039.

Ghosh, T., Chattopadhyay, K., Marschall, M., Karmakar, P. \& Mandal, P. (2009). Focus on antivirally active sulfated polysaccharides: from structure-activity analysis to clinical evaluation. Glycobiology 19: 2-15. 
Hills, S.L. \& Phillips, D.C. (2009). Past, present, and future of Japanese encephalitis. Emerging Infectious Diseases 15: 1333.

Ichiyama, K., Gopala Reddy, S.B., Zhang, L.F., Chin, W.X. \& Muschin, T. (2013). Sulfated polysaccharide, curdlan sulfate, efficiently prevents entry/fusion and restricts antibody-dependent enhancement of dengue virus infection in vitro: a possible candidate for clinical application. PLoS Neglected Tropical Diseases 7: e2188.

Jiao, G., Yu, G., Zhang, J. \& Ewart, H.S. (2011). Chemical structures and bioactivities of sulfated polysaccharides from marine algae. Marine Drugs 9: 196-223.

Lian, W., Wu, M., Huang, N., Gao, N. \& Xiao, C. (2013). Anti-HIV-1 activity and structure-activity-relationship study of a fucosylated glycosaminoglycan from an echinoderm by targeting the conserved CD4 induced epitope. Biochimica et Biophysica Acta 1830: 4681-4691.

Mackenzie, J.S., Gubler, D.J. \& Petersen, L.R. (2004). Emerging flaviviruses: the spread and resurgence of Japanese encephalitis, West Nile and dengue viruses. Nature Medicine 10: S98-109.

Rothan, H.A., Abdulrahman, A.Y., Sasikumer, P.G., Othman, S. \& Rahman, N.A. (2012). Protegrin-1 inhibits dengue NS2B-NS3 serine protease and viral replication in MK2 cells. Journal of Biomedicine and Biotechnology 2012: 251482.

Rothan, H.A., Buckle, M.J., Ammar, Y.A., Mohammadjavad, P. \& Shatrah, O. (2013). Study the antiviral activity of some derivatives of tetracycline and nonsteroid anti inflammatory drugs towards dengue virus. Tropical Biomedicine 30: 681-690.

Rothan, H.A., Mohamed, Z., Suhaeb, A.M., Rahman, N.A. \& Yusof, R. (2013). Antiviral cationic peptides as a strategy for innovation in global health therapeutics for dengue virus: high yield production of the biologically active recombinant plectasin peptide. OMICS 17: 560-567.
Rothan, H.A., Bahrani, H., Mohamed, Z., Abd Rahman, N. \& Yusof, R. (2014). Fusion of protegrin-1 and plectasin to MAP30 shows significant inhibition activity against dengue virus replication. $P L O S$ One 9: e94561.

Rothan, H.A., Zulqarnain, M., Ammar, Y.A., Tan, E.C. \& Rahman, N.A. (2014). Screening of antiviral activities in medicinal plants extracts against dengue virus using dengue NS2B-NS3 protease assay. Tropical Biomedicine 31: 286-296.

Rothan, H.A., Bahrani, H., Mohamed, Z., Teoh, T.C. \& Shankar, E.M. (2015). A combination of doxycycline and ribavirin alleviated chikungunya infection. PLoS One 10: e0126360.

Rothan, H.A., Bidokhti, M.R.M. \& Byrareddy, S.N. (2018). Current concerns and perspectives on Zika virus co-infection with arboviruses and HIV. Journal of Autoimmune 89: 11-20.

Rothan, H.A., Zhong, Y., Sanborn, M.A., Teoh, T.C. \& Ruan, J. (2019). Small molecule grp94 inhibitors block dengue and Zika virus replication. Antiviral Research 171: 104590.

Schmidt, A.G., Yang, P.L. \& Harrison, S.C. (2010). Peptide inhibitors of flavivirus entry derived from the $\mathrm{E}$ protein stem. Journal of Virology 84: 12549-12554.

Smit, J.M., Moesker, B., Rodenhuis-Zybert, I. \& Wilschut, J. (2011). Flavivirus cell entry and membrane fusion. Viruses $\mathbf{3}$ : 160-171.

Spillmann, D. (2001). Heparan sulfate: anchor for viral intruders? Biochimie 83: 811817.

Wang, Q.Y. \& Shi, P.Y. (2015). Flavivirus Entry Inhibitors. ACS Infectious Diseases 1: 428-434.

Wang, H. \& Liang, G. (2015). Epidemiology of Japanese encephalitis: past, present, and future prospects. Therapeutic and Clinical Risk Management 11: 435-448. 